\title{
Medicine in Society
}

\section{J. Grimley Evans}

\begin{abstract}
P. W. F. Wilson, R. J. Garrison and W. P. Castelli, 'Postmenopausal estrogen use, cigarette smoking and cardiovascular morbidity in women over 50: the Framingham study'. New England Journal of Medicine, 313 (1985), 1038-1043.

M. J. Stampfer,W. C. Willett,G. A. Colditz,B. Rosner,F. E. Speizer and C. H. Hennekens, 'A prospective study of postmenopausal estrogen therapy and coronary heart disease'. New England Journal of Medicine, 313 (1985), 1044-1049.
\end{abstract}

The ${ }_{24}$ October 1985 issue of the $\mathcal{N}$ ew England Journal of Medicine carried these two reports of major epidemiological studies of the relationship between postmenopausal use of oestrogens and the risk of cardiovascular disease. The two studies had exactly opposite results. In the first, oestrogen use was associated with a doubling of the risk of cardiovascular disease; in the second oestrogen use was associated with a halving of risk. The conflict of research results raises some worrying reflections about the fallibility of the basis on which policies for both bedside and public health practice are developed.

No obvious reason for the discrepancy in results is apparent. The Framingham study, the most famous and longest-running of epidemiological studies, enrolled some two-thirds of its township in the late I940s and the present report relates to eight years of follow-up of the 1234 postmenopausal women aged 50 and over at the time of the twelfth biennial examination in $197^{\circ-2}$. Of these $24 \%$ reported oestrogen use at some time during the period covered by biennial examinations 8 to 12. Age-adjusted relative risk among oestrogen-users for death from all causes was 0.97 , for death from cancers 0.70 and for death from cardiovascular disease 1.94 - none of these relative risks being significantly different from unity. Relative risks for cardiovascular disease events, that is including non-fatal episodes, were significantly raised at 2.27 for cerebrovascular disease and 1.90 for coronary heart disease. This association of oestrogen use with higher than average cardiovascular disease risk remained when a variety of statistical manipulations to remove the effects of confounding variables was employed.

The second report was of a postal follow-up study of 122,000 nurses originally contacted in 1976 . Of the postmenopausal women $53 \%$ had used oestrogens at some time and $35 \%$ were current users. Age-adjusted relative risk of coronary heart disease in oestrogen users was $0.5(95 \%$ confidence limits $0.3-0.8$ ). Again the difference from unity remained after adjustment for confounding variables. 
Attitudes to the menopause and its management are culturally determined. In Western societies most women regard it as signalling the onset of senility although in the evolutionary perspective it is most likely to have been an evolved response to ageing, specific to the human, enabling women to concentrate on contributing to the survival of their grandchildren rather than wasting their biological resources on increasingly unsuccessful attempts at producing more children of their own. Some feminists, adopting the undoubtedly over-simplified view that the postmenopausal state differs from the premenopausal only in the reduction of oestrogens, have ascribed caution on the part of (male) doctors in prescribing oestrogens as a conspiracy to punish women for their sexual exploitation of men. Doctors have defended themselves by pointing to the increased risk of endometrial cancer associated with oestrogen use and to reasonable fears that oestrogens might also cause breast cancer and cardiovascular disease. Some also doubt whether the effect of oestrogen on perimenopausal symptoms is a pharmacological or placebo response, although this consideration should be less relevant to the practicalities of clinical medicine than some doctors acknowledge. There is certainly, in Britain at least, a tendency for male doctors to underestimate the physical and psychological suffering some women endure at the time of the menopause (an age at which many other sources of stress may be active) and to be too ready to attribute symptoms to the menopause that are due to other remediable causes. These conflicting results from America are therefore thrown into an arena where science, emotion, sexual prejudice and a great deal of ignorance are locked in confused contention.

As an accompanying editorial ${ }^{1}$ points out, the likely response of the scientific community to these findings is a request for further research; it usually is. It could however be argued that since the results are so disparate it is unlikely that oestrogens have a consistent major causative relation with vascular disease and the differences between the two studies reflect differences in the study populations and the ways in which women in the two populations were selected for oestrogen therapy. (The lack of any overall increase in total deaths among oestrogen users in Framingham is also comforting.) There was certainly not enough known about the study groups to adjust for all possible confounding variables, and in the epidemiology of later life it may be that some of the most important confounding variables were acting many years before and could not even be validly assessed in short-term studies. The problem of the generalisability of population-based studies has received in general little attention; scientists, still influenced by laboratory-based models of thought, have perhaps expected too much of the Central Limit 
Theorem and the size of study samples, since the results from a single epidemiological or sociological study can also be regarded as reflecting a sample size of one. On theoretical grounds one might anticipate that differences between populations in changing societies may be greater in older age-groups than at younger.

NOTE

I Bailar, J. G. When research results are in conflict. New England Journal of Medicine, 313 (1985), 1080-1081.

G. S. Rai, P. Murphy and R. A. Pluck, 'Who should provide hospital care of elderly people?' Lancet I (1985), 683-685.

In a specific sense this report raises again the perennial and rather parochial problem of the relation between the specialities of geriatric medicine and of general medicine. More globally, the issue relates to whether or not the elderly benefit as much from specialised, separate services as they could from sharing the same services as other adult age groups. The paper claims that over a period when the geriatric department at a particular London Hospital took an increasing proportion of elderly people into its service, the proportion of elderly medical patients staying in hospital more than 9o days fell from 5.0 to $3.5 \%$ and this was entirely due to a fall from 14.7 to $4.9 \%$ in the department of geriatric medicine while the proportion remained at around $3.0 \%$ among the old people admitted to general medical departments. The study was essentially retrospective and no data were presented on the nature of the cases going to the two services which may well have changed over the study period. There is no evidence that reduced length of stay necessarily represents better care, however welcome it might be to health service administrators. The only value of the paper is as an hypothesis-generating rather than an hypothesis-testing exercise.

An accompanying unsigned editorial comments ${ }^{1}$ that there has never been a controlled trial to test the claim of British geriatricians that they look after the elderly better than do doctors in other hospital specialities. It certainly cannot be assumed that the gross neglect of the elderly that characterised British medicine at the outset of the National Health Service, and which is claimed as the rationale for the creation of geriatric medicine as a speciality, is still as widely prevalent as it was then. In addition to doubts about the quality of their resources, there is also growing anxiety that the existence of separate geriatric depart- 
ments may inhibit the access of old people to the best of 'high technology' medicine which, as data from America (where rationing of medical care is on the basis of ability to pay rather than age) increasingly indicate, can benefit the old as much as the young. Some of the geriatricians most in the public eye seem still to be working to a forty-year old agenda focused on the upgrading of workhouses rather than welcoming the elderly into a share of the good things of the 1980 . Such an agenda is congenial to a government anxious to restrain expenditure on health.

What is at its most basic level merely a squabble within the medical profession has of course wider implications for strategy in social policy for ageing populations. The present British model of specialised services and age-related privileges with its corollary of parallel development may not be preferable to a more radical adaptation of all social institutions and facilities to accommodate the changing distribution of population needs. This is the philosophy, presumably, that leads the editorialist to ask whether rather than diverting more resources into geriatric departments as Rai and colleagues suggest there should be obligatory training in geriatric medicine for all doctors who will eventually have responsibility for elderly patients. Subsequent correspondence in the Lancet vehemently discussed the first suggestion but ignored the second, probably significantly, for it threatens to compromise both the arcana of geriatric medicine and the exclusiveness of other specialities.

NOTE

I Editorial. Geriatrics for all? Lancet, I ( 1985$), 674-675$.

Radcliffe Infirmary, University of Oxford 